\title{
A New Approach Correlating Binaural Hearing and the Brain's Response
}

\author{
Sami Azam ${ }^{\mathrm{a}, *}$, Mirjam Jonkman ${ }^{\mathrm{b}}$, and Friso De Boer ${ }^{\mathrm{c}}$ \\ School of Engineering and IT, Charles Darwin University (CDU), Darwin, Australia \\ E-mail: asami.azam@cdu.edu.au (Corresponding author), bmirjam.jonkman@cdu.edu.au, \\ cfriso.deboer@cdu.edu.au
}

\begin{abstract}
Normal binaural hearing allows the auditory system to determine the direction and distance of sound sources and to detect certain sounds at much lower intensity levels. Different stimuli may have different impact on binaural processing and may generate different brain responses. The mechanism by which this occurs is poorly understood.

Time averaged EEG responses of normal hearing subjects to repeated stimuli were analyzed. The stimuli, $500 \mathrm{~Hz}$ Blackman windowed pure tones, were presented as homophasic or anti-phasic and were also mixed with various noise conditions. Auditory evoked potentials (AEP) were obtained by averaging 500 trials of in-phase and 500 trials of outphase of each EEG epoch.

The results show that the amplitude of the dominant frequency component in the 20 - $50 \mathrm{~Hz}$ range of the middle latency response of the AEP was larger for the anti-phasic condition than for the homo-phasic condition. The normalised amplitude differences were larger when the stimuli were embedded in noise resulting in a higher mean value of the normalized amplitude difference than for noise free stimuli. These results are likely to relate to binaural masking level difference which finds that the detection of a signal in a background noise is easier when the signal has a different inter-aural phase difference than the noise.
\end{abstract}

Keywords: Auditory Evoked Potential (AEP), middle latency response (MLR), homophasic, antiphasic, binaural hearing, binaural masking level difference (BMLD), EEG, stimuli.

ENGINEERING JOURNAL Volume 21 Issue 7

Received 9 July 2017

Accepted 30 August 2017

Published 29 December 2017

Online at http://www.engj.org/

DOI:10.4186/ej.2017.21.7.403 


\section{Introduction}

In a busy acoustical environment it is remarkable how humans can selectively attend to individual sources of sound. Using both ears, the brain behaves as an arithmetic unit tuning to a particular sound source of interest and reduces irrelevant noise from the surrounding environment. It is the 'ability to focus one's attention on a single speaker among a cacophony of conversations and background noise'. This effect is often referred to as 'cocktail party processing' [1] . Analogous to a cocktail party where many conversations are present, our binaural hearing system enables us to tune to an individual conversation and improve speech intelligibility in a noisy environment.

The term 'binaural' refer to the simultaneous use of two ears. The human binaural hearing and the auditory system of the brain system facilitates our ability to detect, localize, separate, and identify sound sources. These tasks are relevant to children who spend many hours in noisy environments such as classrooms and adults who have to operate in similarly complex situations in the work place or in their everyday interactions. Unfortunately, otitis media or middle ear infection is a common disease in childhood that can adversely affect the ability to hear normally [2]. Aboriginal infants and children residing in remote communities and townships often develop chronic ear infections, which leads to hearing and speech disorders in their adult life. This may lead to long lasting conductive hearing loss if left unattended [3-5]. It has been suggested that the conductive hearing loss due to otitis media causes an imbalance in the sound perception in both ears [6]. As a consequence binaural hearing (hearing with both ears), i.e. the auditory processing by the brain involved in the comparison of the sounds received by one ear with the sounds received by the other ear, may be impaired. To understand the impact of binaural hearing on the auditory processing of the brain it is therefore important to investigate the relationship between binaural hearing and brain signals.

The brain's varying electrical potential measured from electrodes placed on the scalp is called the electroencephalogram or EEG [7] . It can be used to diagnose various neurological disorders or for research purposes to gain a better understanding of the human brain. The EEG is used as the primary technique in this research to investigate how electrical activity of the brain responds to binaural stimuli. Electroencephalography is a non-invasive measurement of the brains electrical activity via electrodes attached to the scalp [7]. The nerve cell or neuron is the basic functional unit of the nervous system and the source of electrical signals which communicates information to and from the brain. It is not possible to monitor the activity of a single neuron from the scalp because as the signal propagates towards the electrode it is attenuated by thick layers of tissue. However when a large number of neurons are activated simultaneously the resultant electric field is sufficiently strong to be measured at the scalp. Attaching two or more electrodes to the scalp, which are connected to an amplifier, will reveal a potential difference which will vary over time. The reading of this variation in voltage is called the Electroencephalogram or EEG [7]. During the recording of the EEG signals, the raw EEG signal may also reflect changes in unrelated electrical activity that occurs at the same time. Unwanted signals, that do not have a cerebral origin, are known as artifacts, sometimes also referred to as noise during the experiment. Common types of artifacts include power line artifacts, and artifacts related to muscle activity such as eye movement, eye blinking and movement of the head. EEG due to muscle artifacts is normally of much higher amplitude than AEPs and they are therefore highly discouraged during EEG recordings. In order to have a good quality EEG signals for analysis it is important that artifacts should be avoided or minimized during the experiment and filtered out during the pre-processing phase.

The collective electrical activity of the cerebral cortex is characterized by oscillatory and repetitive behaviour and usually referred to as a rhythm. The EEG signal has the following characteristics [8]:

- The amplitude can vary between 0.5 and 100 micro volts.

- The frequency ranges from 0.5 and $100 \mathrm{~Hz}$.

- The raw EEG is continuous in time.

The amplitude of the EEG signal may vary based on the placement of the scalp electrodes and is also highly dependent on the potential distribution over the scalp surface. It is important to note that scalp potentials depend on the nature and the location of the underlying current sources as well as the conductive and geometric properties of the head [9]. EEG signal measurements have been standardized to a maximum of 124 discrete locations on the scalp surface. In practice, due to time constraints and lack of hardware support, standardised recording techniques limit the number of scalp electrodes to 16 or 32 [10].

The field of auditory evoked potentials comes under the term event related potentials or ERPs. The term ERP can be described as a small variation in voltage which is time locked to a stimulus onset (e.g. sound or image) [9]. The stimulus for an auditory event may be presented via a headphone while a visual stimulus can 
be displayed on a computer monitor. In order to isolate the component of the EEG related to the stimulus, many trials need to be repeated and averaged. The averaging technique is commonly used to improve the signal to noise ratio before any further processing and analysis is performed. The resulting evoked potentials from the auditory stimulation have important clinical applications. Measurements of amplitude and latency are extracted from the averaged evoked potential, which can be used to compare the impact of different sets of stimuli. Testing of the hearing system using AEPs allows for more accurate testing and is independent of an individual's voluntary response. Auditory evoked potentials (AEPs) are classified by the investigated poststimulus time window, distinguishing short latency (brainstem) components (BAEP: 0-10ms), middle latency components (MLAEP: 20-100ms) and long latency components (LLAEP: 100- 500ms) [11].

This study is concerned with conducting a comparative analysis of the effect of $500 \mathrm{~Hz}$ binaural homophasic and antiphasic stimuli embedded with and without noise in the AEP found in the MLR $20 \mathrm{~ms}$ to $100 \mathrm{~ms}$ post stimulation generated. The choice of $500 \mathrm{~Hz}$ frequency for the stimuli depends on a number of factors. $500 \mathrm{~Hz}$ frequency stimuli is in the normal hearing range and are widely used in the standardized hearing tests [12-14]. In addition, 500 frequency stimuli are commonly used to evaluate the auditory pathway [15-20]. By introducing noise to the stimuli, a context is established to the pure tone such as when interpreting speech surrounded by background noise. In addition by introducing noise as a masker to the signal, a binaural masking level difference can be created. The binaural masking level difference (BMLD) is an important psychoacoustic phenomenon that demonstrates and facilitates the use of two ears in detection of signals in noise. It occurs when the cues are presented with interaural level and phase differences [21-23].

\section{Methodology}

\subsection{Normal Hearing Participants}

Normal hearing subjects in the age range of 18 to 35 are selected for this research. Each subject completed a questionnaire to indicate any current or pre-existing conditions which may affect hearing performance. A pure tone audiometric hearing test was used to evaluate the hearing thresholds for each subject. The hearing test was designed to conform to the relevant Australian Standards [12-14]:

- AS IEC 60645-1:2000 Electroacoustics - Audiological Equipment (Part 1: Pure-Tone Audiometers);

- AS/NZS 1269.4:2005 Occupational Noise Management (Part 4: Auditory Assessment).

- AS 1259.1 -1990 Sound level Meters Part 1: Non-integrating.

The environment for the conduction of the test is also governed by the Australian Standards. The maximum allowable background noise level for audiometric testing was below $25 \mathrm{~dB}$ as measured on the sound level meter. This ensures that the soundproof facility is acceptable for an audiometric test and the extraphysiologic artifacts due to the acoustical environment are maintained to a tolerable level.

\subsection{Hardware and Subject Preparation}

EEG data was captured using the process illustrated in Fig. 1. EEG data was recorded from the cortical position $\mathrm{Cz}$ by gold plated electrodes. The measured potentials were sampled at $19.2 \mathrm{kHz}$ using a G.Tec USB biosignal amplifier (Guger Technologies OG, Austria). The G.Tec amplifier has a 24-bit resolution with simultaneous sampling of all channels. It consists of four blocks with four channels and a ground and reference for each block to eliminate the interference from each recorded signal. The audio stimulus was presented with a Creative SoundBlaster Audigy 4 soundcard and AKG-K271 circumaural headphones. The AKG-K271 headphones were calibrated at a sound pressure level of $60 \mathrm{~dB}$.

Twelve participants selected for the study gave an hour of their time for the experiment. Participants were fitted with a head cap with the international 10-20 system electrode placement [9] and asked to sit in a soundproof laboratory. The lights in the laboratory are also switched off in order to reduce $50 \mathrm{~Hz}$ artifacts. Participants were also asked to keep their eye movements minimum. Scalp-electrode impedance levels were kept below $5 \mathrm{k} \Omega$ to prevent poor quality EEG data [24]. 


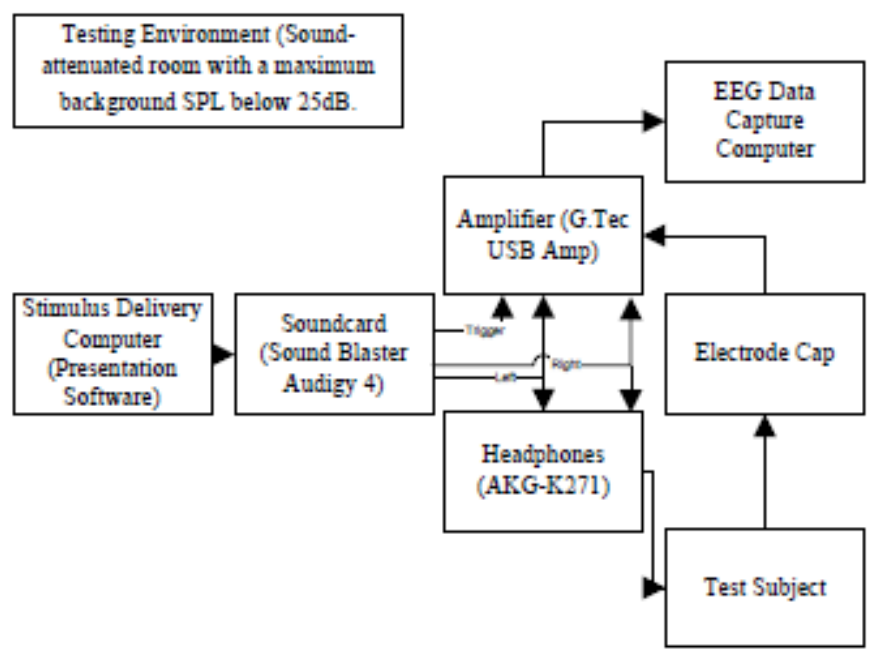

Fig. 1. Hardware and Software setup for EEG recording.

\subsection{Auditory Stimuli and Electrode Locations}

In total, twelve subjects participated in the experiments. Three different types of stimuli were used. All experiments use stimuli consisting of Blackman windowed pure tones of $500 \mathrm{~Hz}$ frequency with a sound pressure level of $60 \mathrm{~dB}$. The stimuli were presented as a block of 10 antiphasic stimuli followed by 10 homophasic stimuli for a total of 1000 trials which provided 500 antiphasic and 500 homophasic related event potentials.

The stimuli for Experiment 2 had noise added to the pure tone. In Experiment 2, the pure tone was masked with continuous Gaussian noise with a bandwidth of 100 to $900 \mathrm{~Hz}$ and the signal to noise ratio for the stimuli was $5 \mathrm{~dB}$. In Experiment 3, the pure tone was again masked with continuous Gaussian noise but with a bandwidth of 20 to $20,000 \mathrm{~Hz}$. The signal to noise ratio for the stimuli was again $5 \mathrm{~dB}$.

The duration of the stimulus in all the experiments was $18 \mathrm{~ms}$. A $200 \mathrm{~ms}$ period of silence between each presentation of stimulus was used to ensure no response of the middle latency response remains at the next stimulus. All experiments presented a total of 1000 stimuli for signal averaging and the auditory evoked potential was measured from the cortical position $(\mathrm{Cz})$ [25] with a reference electrode placed on the left earlobe and ground electrode located on the forehead [26]. The stimuli used for the experiments are summarized in Table 1.

\section{Signal Processing}

\subsection{Processing of Experiment 1, 2 and 3}

The auditory evoked response in the EEG signal from $20 \mathrm{~ms}$ to $100 \mathrm{~ms}$ post stimulation were extracted to look into the middle latency response of the brain elicited by homophasic and antiphasic stimuli. Previous research demonstrated that, contrary to the ABR, its response can be used as an objective electrophysiological measure of low frequency hearing [19,27], while being less time consuming and less affected by difference in attention than the LLR. Epochs which contained significant artefacts were rejected, epochs which contained absolute amplitudes in excess of $\pm 150 \mu \mathrm{V}$ were excluded from the analysis. The remaining epochs are averaged to obtain a good SNR. A Fourier analysis was performed on the averaged epochs for both the antiphasic and homophasic MLR. Initially the first four peaks from the frequency spectrum of the averaged EEG epochs based on antiphasic and homophasic condition were selected. Experimental 1 data were selected in this case to look into the first four spectral peaks. Table 2 and 3 show the amplitude and the frequency of the first four spectral peaks extracted from the frequency spectrum of all 12 subjects' averaged EEG epoch for both antiphasic and homophasic conditions. 
Table 1. Stimuli of Experiment 1,2 and 3.

\begin{tabular}{|c|c|}
\hline \multirow{2}{*}{\multicolumn{2}{|c|}{ Stimuli - $\mathbf{5 0 0 ~ H z ~ F r e q u e n c y ~}$}} \\
\hline & \\
\hline Number of Subjects & 12 \\
\hline Pure tone & Frequency $-500 \mathrm{~Hz}$ \\
\hline Pure tone duration & $18 \mathrm{~ms}$ \\
\hline Silent Duration & $200 \mathrm{~ms}$ \\
\hline Windowing Technique & Blackman Window \\
\hline Sound Pressure Level & $60 \mathrm{~dB}$ \\
\hline Single Trial Duration & $218 \mathrm{~ms}$ \\
\hline Number of trials & 1000 \\
\hline Sequence of Trials & $\begin{array}{l}\text { Blocks of } 10 \text { antiphasic stimuli (180 degree out } \\
\text { of phase) and blocks of } 10 \text { homophasic stimuli } \\
\text { (in same phase), } 50 \text { times each. }\end{array}$ \\
\hline \multicolumn{2}{|c|}{ Experiments 2 and 3} \\
\hline Number of Subjects & 12 \\
\hline Pure tone & Frequency $-500 \mathrm{~Hz}$ masked with noise \\
\hline Pure tone duration & $18 \mathrm{~ms}$ \\
\hline Silent Duration & $200 \mathrm{~ms}$ \\
\hline Windowing Technique & Blackman Window \\
\hline Sound Pressure Level & $60 \mathrm{~dB}$ \\
\hline Single Trial Duration & $218 \mathrm{~ms}$ \\
\hline Number of trials & 1000 \\
\hline Sequence of Trials & $\begin{array}{l}\text { Blocks of } 10 \text { antiphasic stimuli (180 degree out } \\
\text { of phase) and blocks of } 10 \text { homophasic stimuli } \\
\text { (in same phase), } 50 \text { times each. }\end{array}$ \\
\hline Noise type & Gaussian Noise \\
\hline Noise Bandwidth for Experiment 2 & $100-900 \mathrm{~Hz}$ \\
\hline Noise Bandwidth for Experiment 3 & $20-20000 \mathrm{~Hz}$ \\
\hline Interaural Phase Delay of noise & 0 \\
\hline Signal to Noise Ratio & $5 \mathrm{~dB}$ \\
\hline
\end{tabular}

Table 2. Amplitude and Frequency of the first 4 peaks (Antiphasic) - Experiment 1.

\begin{tabular}{lcccccccc}
\hline \multirow{3}{*}{ Subject } & Peak 1 & Peak 2 & Peak 3 & Peak 4 & Peak 1 & Peak 2 & Peak 3 & Peak 4 \\
\cline { 2 - 9 } & $\begin{array}{c}\text { A1Out } \\
{[\mu \mathrm{V}]}\end{array}$ & $\begin{array}{c}\text { A2Out } \\
{[\mu \mathrm{V}]}\end{array}$ & $\begin{array}{c}\text { A3Out } \\
{[\mu \mathrm{V}]}\end{array}$ & $\begin{array}{c}\text { A4Out } \\
{[\mu \mathrm{V}]}\end{array}$ & $\begin{array}{c}\text { F1Out } \\
{[\mathrm{Hz}]}\end{array}$ & $\begin{array}{c}\text { F2Out } \\
{[\mathrm{Hz}]}\end{array}$ & $\begin{array}{c}\text { F3Out } \\
{[\mathrm{Hz}]}\end{array}$ & $\begin{array}{c}\text { F4Out } \\
{[\mathrm{Hz}]}\end{array}$ \\
\hline 1 & 0.7907 & 0.1397 & 0.2540 & 0.1440 & 8.5333 & 23.4667 & 36.2667 & 48.0000 \\
2 & 0.2641 & 0.2827 & 0.2074 & 0.0872 & 8.5333 & 20.2667 & 36.2667 & 51.2000 \\
3 & 0.3207 & 0.3492 & 0.1067 & 0.0913 & 8.5333 & 24.5333 & 51.2000 & 60.8000 \\
4 & 0.2383 & 0.4460 & 0.2061 & 0.3120 & 10.6667 & 26.6667 & 42.6667 & 54.4000 \\
5 & 0.6044 & 0.3132 & 0.2473 & 0.1366 & 8.5333 & 23.4667 & 36.2667 & 50.1333 \\
6 & 0.9029 & 0.2857 & 0.1752 & 0.1455 & 9.6000 & 24.5333 & 39.4667 & 57.6000 \\
7 & 0.9564 & 0.5153 & 0.1189 & 0.2455 & 8.5333 & 24.5333 & 39.4667 & 51.2000 \\
8 & 0.7970 & 0.3307 & 0.2156 & 0.1888 & 10.2667 & 20.2667 & 54.4000 & 73.6000 \\
9 & 0.3025 & 0.1968 & 0.1400 & 0.0972 & 16.0000 & 46.9333 & 61.8667 & 76.8000 \\
10 & 0.4961 & 0.2477 & 0.1906 & 0.1580 & 8.5333 & 35.2000 & 52.2667 & 62.9333 \\
11 & 0.6366 & 0.4052 & 1.1821 & 0.2215 & 17.0667 & 32.0000 & 49.0667 & 66.1333 \\
12 & 1.3171 & 0.7373 & 0.5628 & 0.1729 & 12.8000 & 29.8667 & 44.8000 & 68.2667 \\
\hline
\end{tabular}


Table 3. Amplitude and Frequency of the first 4 peaks (Homophasic) - Experiment 1.

\begin{tabular}{lcccccccc}
\hline \multirow{3}{*}{ Subject } & Peak 1 & Peak 2 & Peak 3 & Peak 4 & Peak 1 & Peak 2 & Peak 3 & Peak 4 \\
\cline { 2 - 9 } & $\begin{array}{c}\text { A1Out } \\
{[\mu \mathrm{V}]}\end{array}$ & $\begin{array}{c}\text { A2Out } \\
{[\mu \mathrm{V}]}\end{array}$ & $\begin{array}{c}\text { A3Out } \\
{[\mu \mathrm{V}]}\end{array}$ & $\begin{array}{c}\text { A4Out } \\
{[\mu \mathrm{V}]}\end{array}$ & $\begin{array}{c}\text { F1Out } \\
{[\mathrm{Hz}]}\end{array}$ & $\begin{array}{c}\text { F2Out } \\
{[\mathrm{Hz}]}\end{array}$ & $\begin{array}{c}\text { F3Out } \\
{[\mathrm{Hz}]}\end{array}$ & $\begin{array}{c}\text { F4Out } \\
{[\mathrm{Hz}]}\end{array}$ \\
\hline 1 & 0.6807 & 0.0884 & 0.2023 & 0.2430 & 8.5333 & 23.4667 & 35.2000 & 51.2000 \\
2 & 0.2782 & 0.2364 & 0.1065 & 0.0274 & 19.2000 & 35.2000 & 50.1333 & 62.9333 \\
3 & 0.0823 & 0.1603 & 0.0887 & 0.0744 & 8.5333 & 32.0000 & 57.6000 & 80.0000 \\
4 & 0.0965 & 0.1594 & 0.1832 & 0.0735 & 6.4000 & 21.3333 & 48.0000 & 70.4000 \\
5 & 0.0525 & 0.2983 & 0.1493 & 0.0653 & 8.5333 & 27.7333 & 49.0667 & 76.8000 \\
6 & 0.7561 & 0.2221 & 0.0933 & 0.1119 & 9.6000 & 26.6667 & 39.4667 & 54.4000 \\
7 & 0.1944 & 0.2413 & 0.2270 & 0.0659 & 9.6000 & 27.7333 & 52.2667 & 75.7333 \\
8 & 1.1056 & 0.2666 & 0.2956 & 0.2567 & 9.6000 & 46.9333 & 60.8000 & 74.6667 \\
9 & 0.3240 & 0.1509 & 0.3406 & 0.1184 & 8.5333 & 33.0667 & 50.1333 & 67.2000 \\
10 & 0.4051 & 0.1708 & 0.1186 & 0.1763 & 14.9333 & 38.4000 & 53.3333 & 68.2667 \\
11 & 0.8817 & 0.3321 & 0.3593 & 0.0901 & 9.6000 & 27.7333 & 52.2667 & 68.2667 \\
12 & 1.2667 & 0.7311 & 0.5004 & 0.3410 & 11.7333 & 28.8000 & 43.7333 & 57.6000 \\
\hline
\end{tabular}

To investigate any difference in the energy carried by the first four spectral peaks of the AEP, the amplitude difference between the spectral peaks are calculated and tabulated as shown in Table 4 below.

Table 4. Amplitude difference of the first 4 peaks - Experiment 1.

\begin{tabular}{lcccc}
\hline Subject & $\begin{array}{c}\text { Peak1 } \\
\text { A1Out- } \\
\text { A1In } \\
{[\mu \mathrm{V}]}\end{array}$ & $\begin{array}{c}\text { Peak2 } \\
\text { A2Out- } \\
\text { A2In } \\
{[\mu \mathrm{V}]}\end{array}$ & $\begin{array}{c}\text { Peak3 } \\
\text { A3Out- } \\
\text { A3In } \\
{[\mu \mathrm{V}]}\end{array}$ & $\begin{array}{c}\text { Peak4 } \\
\text { A4Out- } \\
\text { A4In } \\
{[\mu \mathrm{V}]}\end{array}$ \\
\hline 1 & 0.1101 & $\mathbf{0 . 0 5 1 3}$ & 0.0518 & -0.0990 \\
2 & -0.0142 & $\mathbf{0 . 0 4 6 2}$ & 0.1008 & 0.0599 \\
3 & 0.2384 & $\mathbf{0 . 1 8 8 9}$ & 0.0180 & 0.0169 \\
4 & 0.1418 & $\mathbf{0 . 2 8 6 6}$ & 0.0229 & 0.2385 \\
5 & 0.5519 & $\mathbf{0 . 0 1 4 9}$ & 0.0981 & 0.0714 \\
6 & 0.1468 & $\mathbf{0 . 0 6 3 6}$ & 0.0819 & 0.0335 \\
7 & 0.7620 & $\mathbf{0 . 2 7 4 0}$ & -0.1081 & 0.1796 \\
8 & -0.3085 & $\mathbf{0 . 0 6 4 1}$ & -0.0800 & -0.0680 \\
9 & -0.0214 & $\mathbf{0 . 0 4 5 9}$ & -0.2007 & -0.0213 \\
10 & 0.0910 & $\mathbf{0 . 0 7 6 9}$ & 0.0720 & -0.0183 \\
11 & -0.2451 & $\mathbf{0 . 0 7 3 0}$ & 0.8228 & 0.1315 \\
12 & 0.0504 & $\mathbf{0 . 0 0 6 2}$ & 0.0624 & -0.1681 \\
\hline
\end{tabular}

Table 4 shows that the amplitude difference between the second spectral antiphasic peak and second spectral homophasic peak have the highest number of positive difference compared to the difference between amplitudes of the first, the third and the fourth antiphasic and homophasic second spectral peaks frequency. Table 5, 6 and 7 below of Experiment 1,2 and 3 respectively show the amplitude and the frequency of the second spectral peaks extracted from the frequency spectrum of all 12 subject's averaged EEG epochs for both antiphasic and homophasic conditions. 
Table 5. Second dominant frequency peaks and amplitude difference - Experiment 1 .

\begin{tabular}{cccccc}
\hline Subject & $\begin{array}{c}\text { Peak 2 } \\
\text { A2Out }\end{array}$ & $\begin{array}{c}\text { Peak 2 } \\
\text { F2Out }\end{array}$ & $\begin{array}{c}\text { Peak 2 } \\
\text { A2In }\end{array}$ & $\begin{array}{c}\text { Peak 2 } \\
\text { F2In }\end{array}$ & $\begin{array}{c}\text { Peak 2 } \\
\text { A2Out- } \\
\text { A2In }\end{array}$ \\
\hline 1 & 0.1397 & 23.4667 & 0.0884 & 23.4667 & $\mathbf{0 . 0 5 1 3}$ \\
2 & 0.2827 & 20.2667 & 0.2364 & 35.2 & $\mathbf{0 . 0 4 6 3}$ \\
3 & 0.3492 & 24.5333 & 0.1603 & 32 & $\mathbf{0 . 1 8 8 9}$ \\
4 & 0.446 & 26.6667 & 0.1594 & 21.3333 & $\mathbf{0 . 2 8 6 6}$ \\
5 & 0.3132 & 23.4667 & 0.2983 & 27.7333 & $\mathbf{0 . 0 1 4 9}$ \\
6 & 0.2857 & 24.5333 & 0.2221 & 26.6667 & $\mathbf{0 . 0 6 3 6}$ \\
7 & 0.5153 & 24.5333 & 0.2413 & 27.7333 & $\mathbf{0 . 2 7 4}$ \\
8 & 0.3307 & 20.2667 & 0.2666 & 46.9333 & $\mathbf{0 . 0 6 4 1}$ \\
9 & 0.1968 & 46.9333 & 0.1509 & 33.0667 & $\mathbf{0 . 0 4 5 9}$ \\
10 & 0.2477 & 35.2 & 0.1708 & 38.4 & $\mathbf{0 . 0 7 6 9}$ \\
11 & 0.4052 & 32 & 0.3321 & 27.7333 & $\mathbf{0 . 0 7 3 1}$ \\
12 & 0.7373 & 29.8667 & 0.7311 & 28.8 & $\mathbf{0 . 0 0 6 2}$ \\
\hline
\end{tabular}

Table 6. Second dominant frequency peaks and amplitude difference - Experiment 2.

\begin{tabular}{lccccc}
\hline Subject & $\begin{array}{c}\text { Peak 2 } \\
\text { A2Out }\end{array}$ & $\begin{array}{c}\text { Peak 2 } \\
\text { F2Out }\end{array}$ & $\begin{array}{c}\text { Peak 2 } \\
\text { A2In }\end{array}$ & $\begin{array}{c}\text { Peak 2 } \\
\text { F2In }\end{array}$ & $\begin{array}{c}\text { Peak 2 } \\
\text { A2Out- } \\
\text { A2In }\end{array}$ \\
\hline 1 & 0.1320 & 29.8667 & 0.0604 & 25.6000 & $\boldsymbol{0 . 0 7 1 6}$ \\
2 & 0.1627 & 30.9333 & 0.0655 & 34.1333 & $\mathbf{0 . 0 9 7 2}$ \\
3 & 0.1661 & 22.4000 & 0.0683 & 35.2000 & $\mathbf{0 . 0 9 7 8}$ \\
4 & 0.1719 & 21.3333 & 0.1441 & 30.9333 & $\boldsymbol{0 . 0 2 7 9}$ \\
5 & 0.3061 & 29.8667 & 0.1656 & 29.8667 & $\boldsymbol{0 . 1 4 0 5}$ \\
6 & 0.1447 & 28.8000 & 0.0600 & 26.6667 & $\boldsymbol{0 . 0 8 4 7}$ \\
7 & 0.2919 & 42.2667 & 0.2020 & 36.2667 & $\mathbf{0 . 0 8 9 9}$ \\
8 & 0.1882 & 30.9333 & 0.1284 & 20.2667 & $\mathbf{0 . 0 5 9 9}$ \\
9 & 0.1593 & 29.8667 & 0.1305 & 29.8667 & $\boldsymbol{0 . 0 2 8 7}$ \\
10 & 0.4267 & 25.6000 & 0.2620 & 46.9333 & $\boldsymbol{0 . 1 6 4 7}$ \\
11 & 0.4422 & 24.5333 & 0.1306 & 45.8667 & $\mathbf{0 . 3 1 1 6}$ \\
12 & 0.2287 & 40.5333 & 0.1819 & 33.0667 & $\mathbf{0 . 0 4 6 9}$ \\
\hline
\end{tabular}

Table 7. Second dominant frequency peaks and amplitude difference - Experiment 3.

\begin{tabular}{lccccc}
\hline Subject & $\begin{array}{c}\text { Peak 2 } \\
\text { A2Out }\end{array}$ & $\begin{array}{c}\text { Peak 2 } \\
\text { F2Out }\end{array}$ & $\begin{array}{c}\text { Peak 2 } \\
\text { A2In }\end{array}$ & $\begin{array}{c}\text { Peak 2 } \\
\text { F2In }\end{array}$ & $\begin{array}{c}\text { Peak 2 } \\
\text { A2Out- } \\
\text { A2In }\end{array}$ \\
\hline 1 & 0.3227 & 24.5333 & 0.2596 & 26.6667 & $\boldsymbol{0 . 0 6 3 1}$ \\
2 & 0.1431 & 36.2667 & 0.0666 & 34.1333 & $\mathbf{0 . 0 7 6 5}$ \\
3 & 0.4286 & 24.5333 & 0.4212 & 27.7333 & $\mathbf{0 . 0 0 7 3}$ \\
4 & 0.3572 & 34.1333 & 0.0815 & 22.4000 & $\mathbf{0 . 2 7 5 7}$ \\
5 & 0.4161 & 32.0000 & 0.3587 & 29.8667 & $\mathbf{0 . 0 5 7 4}$ \\
6 & 0.2665 & 28.8000 & 0.1900 & 25.6000 & $\boldsymbol{0 . 0 7 6 5}$ \\
7 & 0.3562 & 24.5333 & 0.2246 & 29.8667 & $\mathbf{0 . 1 3 1 6}$ \\
8 & 0.1644 & 34.1333 & 0.1227 & 43.7333 & $\mathbf{0 . 0 4 1 8}$ \\
9 & 0.0566 & 37.3333 & 0.0189 & 36.2667 & $\mathbf{0 . 0 3 7 7}$ \\
10 & 0.4210 & 28.8000 & 0.3291 & 29.8667 & $\boldsymbol{0 . 0 9 1 9}$ \\
11 & 0.1906 & 32.0000 & 0.1845 & 29.8667 & $\boldsymbol{0 . 0 0 6 1}$ \\
12 & 0.5058 & 27.7333 & 0.5046 & 29.8667 & $\mathbf{0 . 0 0 1 2}$ \\
\hline
\end{tabular}


As seen from Table 5 to 7 there is a clear positive amplitude difference between the antiphasic and homophasic AEPs of the MLR in the second spectral frequency peaks. All the subjects for each experiment showed higher second spectral peak amplitude when presented with antiphasic stimuli. It is the second spectral peak found in the frequency range of 20 to $50 \mathrm{~Hz}$ where all the subjects in the study showed higher second spectral peak amplitude when presented with antiphasic stimuli. The positive amplitude apparent in the tables indicates that antiphasic stimuli yield a higher second dominant frequency peak in the spectrum of the averaged AEP in the middle latency of the brain.

\subsection{Comparison between Experiments - Second Spectral Peak Amplitude}

Further analysis of the results is conducted in order to compare between the experiments to find the difference in the impact of different stimuli. In this case, the second spectral peak amplitude difference for each subject under each experiment was divided by the average of second spectral homophasic and antiphasic peak amplitudes. The purpose of dividing with the average value of homophasic and antiphasic spectral peak amplitude was to normalize the amplitude difference of the second spectral peaks by including a division with the overall impact of the second spectral dominant EEG frequencies. This may account for variability in amplitudes of subjects or the experimental conditions of that particular experiment.

Once the second spectral frequency peak difference was normalized a mean value of all the normalized amplitude differences for each experiment was calculated and a comparison between the experiments was conducted based on the magnitude of the mean value achieved for each experiment. The formula to normalize the amplitude difference and to calculate the mean value of all the 12 normalised amplitude differences under each experiment is shown in Eq. (1).

$$
\text { Mean }=\frac{1}{12} * \sum_{i=1}^{12} \frac{\left(A 2: O U T_{i}-A 2: I N_{i}\right)}{\left(A 2: O U T_{i}+A 2: I N_{i}\right) * \frac{1}{2}}
$$

Table 8 below shows the normalized and the mean value of the second spectral frequency peak amplitude difference for all the subjects of Experiment 1,2 and 3.

Table 8. Normalised amplitude difference for Experiment 1, 2 and 3.

\begin{tabular}{|c|c|c|c|}
\hline & $\begin{array}{l}\text { Experiment } 1 \\
10 \text { Block Out - } 10 \\
\text { Block In }\end{array}$ & $\begin{array}{l}\text { Experiment } 2 \\
10 \text { Block Out }-10 \\
\text { Block In }(100 \mathrm{~Hz} \\
\text { to } 900 \mathrm{~Hz}-\text { masked } \\
\text { noise) }\end{array}$ & $\begin{array}{l}\text { Experiment } 3 \\
10 \text { Block Out }-10 \\
\text { Block In ( } 20 \mathrm{~Hz} \text { to } \\
20000 \mathrm{~Hz}-\text { masked } \\
\text { noise) }\end{array}$ \\
\hline Subject & AmpDiff/AvgAmp & AmpDiff/AvgAmp & AmpDiff/AvgAmp \\
\hline 1 & 0.4495 & 0.7447 & 0.2166 \\
\hline 2 & 0.1781 & 0.8519 & 0.7294 \\
\hline 3 & 0.7417 & 0.8342 & 0.0173 \\
\hline 4 & 0.9467 & 0.1764 & 1.2571 \\
\hline 5 & 0.0486 & 0.5955 & 0.1482 \\
\hline 6 & 0.2505 & 0.8273 & 0.3352 \\
\hline 7 & 0.7244 & 0.3640 & 0.4530 \\
\hline 8 & 0.2146 & 0.3781 & 0.2910 \\
\hline 9 & 0.2639 & 0.1982 & 0.9987 \\
\hline 10 & 0.3676 & 0.4783 & 0.2451 \\
\hline 11 & 0.1980 & 1.0879 & 0.0325 \\
\hline 12 & 0.0084 & 0.2283 & 0.0023 \\
\hline MEAN & 0.3660 & 0.5637 & 0.3939 \\
\hline
\end{tabular}


A graphical representation of the mean normalised amplitude difference for experiment 1,2 and 3 is shown in the Fig. 2.

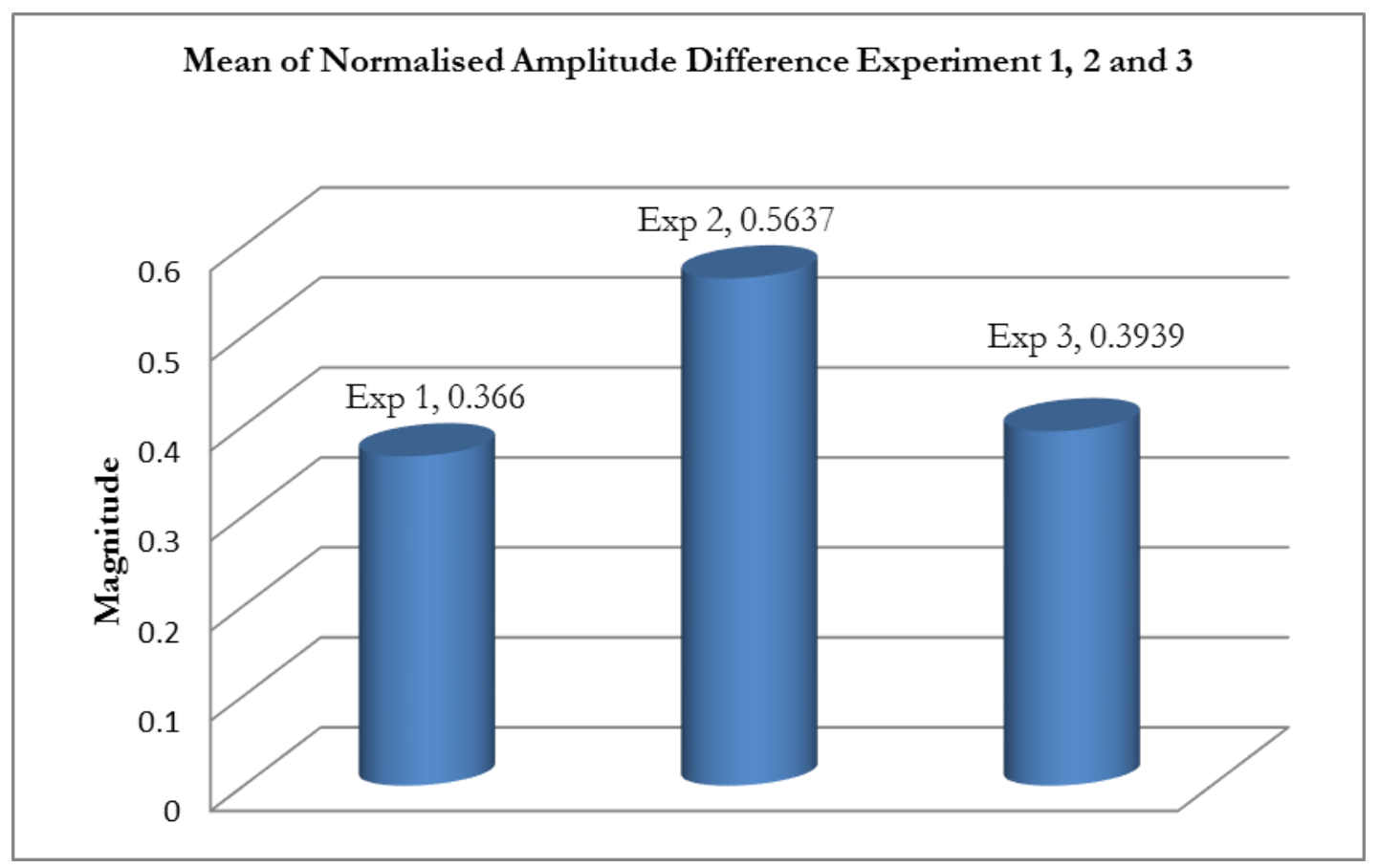

Fig. 2. Mean of normalised amplitude difference final experiment 1,2, 3.

Experiment 2 yields the largest mean of the normalized amplitude difference compared to the other two experiments. In Experiment 2, the Blackman windowed $500 \mathrm{~Hz}$ pure tone stimulus was masked with noise with a bandwidth of $100 \mathrm{~Hz}$ to $900 \mathrm{~Hz}$. The second largest difference is found for Experiment 3 where the stimulus was masked by noise with a bandwidth of $20 \mathrm{~Hz}$ to $20 \mathrm{kHz}$. Experiment 1, where stimuli that were not masked with noise were applied, yields the smallest difference. These results possibly indicate that a narrow band noise masker acts as a cue for the subject to perceive tones better in noise, thus eliciting a larger mean value of normalized amplitude difference in the second spectral peak frequency component.

\section{Results and Discussions}

After analysing the experimental data it is found that it is the second spectral peak that lies in the frequency band of around 20 to $50 \mathrm{~Hz}$ can be used to find the difference in auditory evoked response elicited by homophasic and antiphasic stimuli. The second spectral peak consistently has a higher amplitude when elicited by antiphasic stimuli. The frequency range of the second peak indicates that this effect possibly relates to the middle latency response (MLR).

The higher second spectral peak amplitude found in the auditory response evoked due to antiphasic stimuli supports Durlach's "Equalization and Cancellation" theory. This agrees with the theory behind Durlach's "Equalization-Cancellation" for the 180 degree phase shift, which says that humans will hear stimuli better when they are presented out of phase [28, 29].

As shown in Table 8 , it is found that a pure tone embedded with noise yields the higher mean value of normalized second spectral peak amplitude differences compared to the values achieved for pure tone with no noise. This suggests that background noise may provide additional binaural cues for binaural processing. By introducing noise to the stimuli, a context is established to the pure tone such as when interpreting speech surrounded by background noise. In addition by introducing noise as a masker to the signal, a binaural masking level difference can be created. It is also reported by Ira and Licklider that the detection of a signal in a background of noise is much easier when the signal has a different inter-aural time or phase difference than that of the noise [22, 23].

Of the three experiments, it is found that the middle latency response elicited by a $500 \mathrm{~Hz}$ pure tone embedded with $100 \mathrm{~Hz}$ to $900 \mathrm{~Hz}$ Gaussian noise yields the maximum mean value of normalized amplitude 
difference. This may be due to an increase in binaural masking level difference with increasing signal interaural level difference, with increasing masker intensity level or with a more narrow masker bandwidth [21].

\section{Conclusions}

Auditory evoked potentials (AEPs) were recorded and analysed to investigate the electrophysiological effect in the neural activity based on the phase reversal of the binaural stimuli. AEPs for both homophasic and antiphasic conditions were obtained by averaging 500 trials of in-phase and 500 trials of out-phases of each EEG epoch. Stimuli consisted of Blackman windowed $500 \mathrm{~Hz}$ pure tones of 18 ms duration, mixed with various noise conditions. The AEPs were then analysed in the frequency domain.

It was found that the amplitude of the second spectral peak of the MLR was larger for the antiphasic condition than the homophasic condition. The peak occurred in the range of $20-50 \mathrm{~Hz}$. The results indicate that the amplitude of the second peak can be used as a marker to detect binaural processing in the human brain. The behavior of the second peak indicates changes in the middle latency response (MLR) as the frequencies of the second peak correspond with this response $(20-50 \mathrm{~Hz})$. The results support Durlach's "Equalization-Cancellation" theory which suggests that humans will hear stimuli better when they are presented out of phase $[28,29]$. It was also found that pure tone stimuli embedded with noise yield higher mean values of normalised second spectral peak amplitude differences than stimuli of pure tones without noise.

The findings of this research are likely to relate the psychoacoustic phenomenon known as binaural masking level difference (BMLD). The overall results indicate that the detection of a signal in a background of noise is much easier when the signal has a different inter-aural phase difference than that of the noise, as was to be expected from literature [22,23].

\section{References}

[1] B. Arons, "A review of the cocktail party effect," Journal of the American Voice I/O Society, vol. 12, pp. 35-57,1992.

[2] C. J. Williams and A. M. Jacobs, "The impact of otitis media on cognitive and educational outcomes," Medical Journal of Australia, vol. 191, pp. S69-S72, 2009.

[3] G. J. McCafferty, A. N. Lewis, W. B. Coman, and C. Mills, "A nine-year study of ear disease in Australian Aboriginal children," J Laryngol Otol, vol. 99, pp. 117-125, 1985.

[4] P. S. Morris, A. J. Leach, P. Silberberg, G. Mellon, C. Wilson, E. Hamiltion, and J. Meissbarth, "Otitis media in young Aboriginal children from remote communities in Northern and Central Australia: A cross-sectional survey," BMC Pediatrics, vol. 5, no. 1, p. 27, 2005.

[5] A. J. Leach, "Otitis media in Australian Aboriginal children: An overview," International Journal of Pediatric Otorbinolaryngology, vol. 49, pp. S173-S178, 1999.

[6] J. Roberts, L. Hunter, J. Gravel, R. Rosenfeld, S. Berman, M. Haggard, J. Hall, C. Lannon, D. Moore, L. Vernon-Feagans, and I. Wallace, "Otitis media, hearing loss, and language learning: controversies and current research," Journal of Developmental and Behavioral Pediatrics, vol. 25, no. 2, pp. 110-122, 2004.

[7] M. Teplan, "Fundamentals of EEG measurement," Measurement Science Review, vol. 2, pp. 1-11, 2002.

[8] A. G. Gaidhani and D. Chuadhari, "Electroencephalogram recording system," in Engineering in Medicine and Biology Society IEEE, 1995, pp. 25-26.

[9] S. K. Law, P. L. Nunez, A. F. Westdorp, A. V. Nelson, and K. L. Pilgreen, "Topographical mapping of brain electrical activity," in Proceedings of the $2^{\text {nd }}$ conference on Visualization'91, IEEE, 1991, pp. 194-201.

[10] A. Gevins, P. Brickett, B. Costales, J. Le, and B. Reutter, "Beyond topographic mapping: Towards functional-anatomical imaging with 124-channel EEGs and 3-D MRIs," Brain topography, vol. 3, pp. 5364, 1990.

[11] Y. Fukui, M. Mori, M. Kohga, T. Tadai, K. Tanaka, and N. Katoh, "Reassessment of CNS effects of acute ethanol administration with auditory evoked response: A comparative study of brain stem auditory evoked response, middle latency response and slow vertex response," Jpn. J. Alcohol Drug Depend, vol. 16, pp. 9-32, 1981.

[12] Electroacoustics - Audiological Equipment Part 1: Pure-Tone Audiometers, Australian Standard, AS IEC 60645$1,2000$. 
[13] Occupational Noise Management Part 4: Auditory Assessment, Australian/New Zealand Standard, AS/NZS 1269.4, 2005.

[14] Sound Level Meters Part 1: Non-integrating, Australian Standard, AS1259.1, 1990.

[15] K. T. Kavanagh, P. L. Crews, W. D. Domico, and V. A. McCormick, "Comparison of the intrasubject repeatability of auditory brain stem and middle latency responses elicited in young children," Annals of Otology, Rhinology and Laryngology, vol. 97, pp. 264-271, 1988.

[16] E. O. Laukli and I. Mair, "Low-frequency auditory brainstem response threshold," Scandinavian Audiology, vol. 17, pp. 171-178, 1988.

[17] T. Dau, O. Wegner, V. Mellert, and B. Kollmeier, "Auditory brainstem responses with optimized chirp signals compensating basilar-membrane dispersion," The Journal of the Acoustical Society of America, vol. $107,2000$.

[18] K. J. Mobley and E. Gibson. (2006). Tone Burst Evoked Potentials: Clinical Applications [Online]. Available: http://www.audiologyonline.com/articles/tone-burst-evoked-potentials-clinical-1279/ [Accessed: 6 June 2017].

[19] N. Kraus and T. McGee, "Clinical applications of the middle latency response," Journal of the American Academy of Audiology, vol. 1, pp. 130-133, 1990.

[20] W. Y. Wong and D. R. Stapells, "Brain stem and cortical mechanisms underlying the binaural masking level difference in humans: An auditory steady-state response study," Ear and Hearing, vol. 25, pp. $57-$ 67, 2004.

[21] W. Y. Wong and D. R. Stapells, "Brain stem and cortical mechanisms underlying the binaural masking level difference in humans: An auditory steady-state response study," Ear and Hearing, vol. 25, pp. 5767, 2004.

[22] R. M. Stern and C. Trahiotis, "Models of binaural perception," Binaural and Spatial Hearing in Real and Virtual Environments, vol. 24, pp. 499-531, 1997.

[23] I. J. Hirsh, "The influence of interaural phase on interaural summation and inhibition," The Journal of the Acoustical Society of America, vol. 20, p. 536, 1948.

[24] H. Riedel and B. Kollmeier, "Comparison of binaural auditory brainstem responses and the binaural difference potential evoked by chirps and clicks," Hearing Research, vol. 169, pp. 85-96, 2002.

[25] H. G. Vaughan and W. Ritter, "The sources of auditory evoked responses recorded from the human scalp," Electroencephalography and Clinical Neurophysiology, vol. 28, pp. 360-367, 1970.

[26] G. Plourde, "Auditory evoked potentials," Best Practice and Research Clinical Anaesthesiology, vol. 20, pp. 129-139, 2006.

[27] J. Nousak and D. R. Stapells, "Auditory brainstem and middle latency responses to $1 \mathrm{kHz}$ tones in noise-masked normally-hearing and sensorineurally hearing-impaired adults," International Journal of Audiology, vol. 44, pp. 331-344, 2005.

[28] M. A. Akeroyd, "The psychoacoustics of binaural hearing," International Journal of Audiology, vol. 45, pp. 25-33, 2006.

[29] M. Heijden and C. Trahiotis, "Binaural detection as a function of interaural correlation and bandwidth of masking noise: Implications for estimates of spectral resolution," The Journal of the Acoustical Society of America, vol. 103, pp. 1609-1614, 1998. 